\title{
ICBR-Diff: an Impairment Constraint Based Routing Strategy with Quality of Signal Differentiation
}

\author{
Amornrat Jirattigalachote, Paolo Monti, Lena Wosinska \\ Royal Institute of Technology KTH, School of Information and Communication Technology, Isafjordsgatan 22, \\ Electrum 229, 16440 Kista, Sweden \\ Email: \{amornrat, pmonti, wosinska\}@kth.se \\ Kostas Katrinis and Anna Tzanakaki \\ Athens Information Technology (AIT), 19.5 Markopoulo Av., P.O. Box 68, 19002 Peania, Greece \\ Email: \{kkat, atza\}@ait.edu.gr
}

\begin{abstract}
Latest advances in Wavelength Division Multiplexing (WDM) technology make it possible to build all-optical transparent networks, which are considered to be able to satisfy the rapidly growing capacity demand. However, in a transparent WDM network the optical signal transmitted from a source to a destination node might be degraded due to physical layer impairments induced by transmission through optical fibers and components. Several Impairment Constraint Based Routing (ICBR) algorithms have been proposed to consider physical layer impairments during the connection-provisioning phase in order to prevent selecting a lightpath with poor signal quality. However, these algorithms support only a single quality of transmission threshold for all connection requests, while next generation networks and the future Internet are expected to support a variety of services with potentially disparate QoS requirements. In this paper, we propose the use of bit error rate (BER) as a differentiation of service parameter for connection requests in optical WDM networks. This is achieved through the use of ICBR, whereby various BER thresholds are set depending on the QoS requirements for accepting/blocking the connection requests during the connection-provisioning phase. The evaluation results reveal that significant network performance improvement in terms of connection blocking can be achieved, compared to non-differentiated conventional routing and wavelength assignment (RWA) and ICBR algorithms.
\end{abstract}

Index Terms-Impairment Constraint Based Routing (ICBR), differentiation of services, signal quality, physical layer impairments, connection provisioning, transparent optical networks.

\section{INTRODUCTION}

Transparent WDM networks constitute a promising solution to cater for the rapid growing of bandwidth

Parts of this paper appeared in Proc. of IEEE ICTON/RONEXT'09; Manuscript received January 29, 2010; accepted March 22, 2010. demand in next generation networks and the future Internet. In such networks, the signal is transported from source to destination in the optical domain via all-optical channels (also referred to as lightpaths [1]) with capacity that can reach $100 \mathrm{Gbit} / \mathrm{s}$ [2]. These lightpaths do not require any intermediate optoelectronic processing, thus reducing the number of costly $\mathrm{O} / \mathrm{E} / \mathrm{O}$ converters. In addition, all-optical networks offer bit rate, signal format, and protocol transparency.

Many routing and wavelength assignment (RWA) algorithms [3][4] have been proposed in order to assign a lightpath, i.e. a route and wavelength, to a connection request. One objective of these RWA algorithms is to reserve the minimum amount of network resources for all connection requests. By minimizing the amount of reserved resources, the reduction in connection blocking, i.e. the blocking of a connection request due to the lack of available resources in the network, is expected.

However, these RWA algorithms base their routing decisions only on the availability of network resources, and assume that optical fibers and components are ideal. But in practice, an optical signal might be degraded by physical layer impairments inherent in the fiber segments and optical components, in the absence of intermediate optoelectronic conversions offering signal regeneration [5]. This effect clearly introduces the need to consider physical layer impairments during the connectionprovisioning phase, i.e. the need to solve the so-called Impairment Aware Routing and Wavelength Assignment (IA-RWA) problem. The objective of solving the IARWA problem is not only to minimize the amount of reserved network resources, i.e. to reduce the connection blocking, but also to guarantee the required quality of transmission level, e.g. measured in terms of bit error rate (BER), for each connection request.

Physical layer impairments can be divided into linear and non-linear [6][7]. Linear impairments do not depend on the signal power and affect each channel individually. A list of the most important linear impairments includes: Amplified Spontaneous Emission (ASE) noise, Group 
Velocity Dispersion (GVD) and Polarization Mode Dispersion (PMD). ASE is due to optical amplification, whereby optical amplifiers degrade the optical signal to noise ratio (OSNR) of the transmitted optical signal. GVD causes a pulse broadening effect and is the result of different spectral components traveling at different velocities. PMD causes optical power variation and signal distortion due to the fiber birefringence where each of the two orthogonally polarized modes travels slower than the other. Non-linear impairments on the other hand, are triggered due to the response of the optical fiber to intense optical signals [8][9]. Specific nonlinear effects that are important in the context of optical signal transmission in fibers are Self Phase Modulation (SPM) and Cross Phase Modulation (XPM) caused by the dependence of the fiber refractive index on the intensity of the applied electromagnetic field. SPM is an effect through which an optical signal causes degradation of itself in the form of spectral broadening and pulse chirping. On the other hand, XPM introduces spectral broadening of the optical signal through its interaction with the other channels also transmitted in the same fiber. Another important nonlinear effect is Four Wave Mixing (FWM), by reason of which unwanted optical components are generated due to propagation of the optical channels through the fiber. Nonlinear effects are in general emphasized for high signal power levels and longer transmission distances. In summary, both linear and non-linear impairments are highly dependent on the fiber characteristics which in turn are sensitive to length, temperature and age.

Several Impairment Constraint Based Routing (ICBR) algorithms [10][11] have been proposed in the literature to solve the IA-RWA problem. The work in [10] presents two different ICBR algorithms. One of them first selects the lightpath with the shortest physical distance and then it checks the lightpath signal quality. The second one picks the first available wavelength on the selected route, and then performs a signal quality check on that specific wavelength. If the signal quality of the selected wavelength does not satisfy the signal quality threshold, the next available wavelength is chosen. Both ICBR algorithms in [10] compare the signal quality of the selected lightpath against a single quality threshold and consider only linear physical layer impairments, namely PMD and ASE noise. The work in [11] considers, in addition to linear physical layer impairments, also the effect of non-linear impairments, i.e. SPM, XPM, and FWM. The authors present an ICBR algorithm that characterizes and orders the wavelengths based on their quality factor (Q-factor) values and it chooses the lightpath with the highest Q-factor. This lightpath quality is then compared against a single quality threshold.

The family of ICBR algorithms outlined above are usually referred to as Impairment-Aware Best-Path (IABP) routing algorithms because of the way they make routing decisions, i.e., they always assign each connection request the least impaired lightpath, regardless of the signal quality requirement of the connection request. However, a variety of services, such as peer-to- peer (P2P) applications, high definition television (HDTV) and Audio Video On Demand (AVOD), require different quality of transmission, bandwidth and delay, while these ICBR algorithms treat all connection requests in the same manner. In other words, they support only a single signal quality threshold, uniformly to all connection requests (i.e. the lowest BER). As a consequence, these single-threshold approaches may unnecessary block connection requests that could sustain signal degradation of some extent, i.e. higher BER than the single threshold imposed by these flat-service ICBR schemes. In addition, for those connection requests that are accepted, such single-threshold approaches may overprovision network resources, i.e. connection requests with low BER threshold are unnecessarily assigned well performing fibers, with a potentially detrimental effect on the overall connection blocking.

To overcome these deficiencies, this paper proposes and evaluates a novel ICBR algorithm, referred to as ICBR-Diff, supporting differentiation of services at the $\mathrm{BER}$ level. In the proposed algorithm, various BER thresholds are considered for accepting/blocking connection requests in the connection-provisioning phase, depending on the signal quality requirements of the connection requests. This allows for a better use of the available resources by offering optical signal quality that is good enough for a specific connection request. Simulation results on the Pan-European test network topology and NSFNet topology, show significant improvement in terms of connection blocking, compared with conventional Shortest Path and IABP algorithms.

The rest of this paper is organized as follows. Section II presents the transmission link model and physical layer impairments model used for proposed algorithm. In Section III, the proposed ICBR-Diff algorithm is described. The performance evaluation of the ICBR-Diff algorithm is presented in Section IV. Finally, Section V provides some concluding remarks.

\section{ESTIMATING THE TRANSMISSION QUALITY OF THE CONNECTION REQUESTS}

This section describes the assumptions made and provides details on (i) the approach and (ii) the equations used to estimate the transmission quality of a connection request routed along a given path in the network.

It is assumed that the WDM network has an arbitrary physical topology (mesh). The WDM mesh is modeled as a graph $G=(N, L)$ where $N$ represents the set of network nodes, i.e. OXCs, and $L$ represent the set of network links. It is assumed that every network link consists of a set of bidirectional fibers, $F$, where each fiber carries a set of wavelengths, $W$.

Given a connection request $r$ between a source and a destination node, the objective of any impairment constrained based routing strategy is to find a path $p \in$ $G=(N, L)$ able to accommodate some predefined quality of transmission requirements. In this work the quality of transmission is measured in terms of bit error rate (BER). The BER computation may vary depending on the assumptions made in modeling a network link and on the 
optical impairments that are considered. A detailed description of the assumptions used to model each network link is provided next.

\section{A. Network link model}

Amplification and dispersion management [12][13] techniques are some of the available approaches used to increase the reach of optical transparent transmissions, and to guarantee an acceptable level of signal quality at the receiver (i.e. the destination node). In this work, these techniques, combined with the approach presented by G. Markidis et al. [11], are used to model the transmission link connecting two nodes in the network, as shown in Fig. 1.

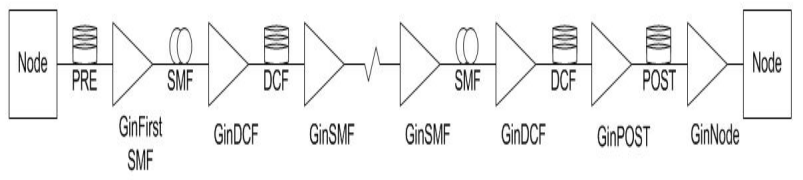

Figure 1. Network link architecture.

Fig. 1 shows that the assumed network link consists of a sequence of single mode fiber (SMF) spans. Their number may vary according to the physical distance separating the two nodes. Erbium-doped fiber amplifiers (EDFAs) are inserted following each fiber span in order to compensate for the power loss induced by the fiber. For long-haul DWDM transmission careful dispersion management is critical. In this context, a non-resonant dispersion map that utilizes pre-compensation and postcompensation aiming to achieve optimum system performance is used. Accumulated dispersion is also compensated by allocating in line dispersion compensating fiber (DCF). Based on this model, the Qfactor of each link is computed. This computation is then be used in the estimation of the transmission quality on an entire path $p$, as explained in the next sections.

\section{B. Estimating the transmission quality of a link in the network}

In this work, the effect of physical layer impairments is quantified using the quality factor $\mathrm{Q}$, defined as [11]. The Q-penalty factor includes both linear and non-linear physical layer impairments, namely ASE noise, the combined effects of SPM/GVD and optical filtering, FWM, and XPM. ASE, FWM and XPM are calculated by assuming that they follow a Gaussian distribution. For the combined SPM/GVD and optical filtering effects, they are quantified through an eye closure penalty metric. The Q-penalty factor on the $k$-th link can be expressed as:

$$
Q_{\text {penalty }, k}=\frac{\sqrt{\sigma_{A S E, k}^{2}+\sigma_{F W M, k}^{2}+\sigma_{X P M, k}^{2}}}{\text { pen }_{\text {eye }, k}},
$$

where pen $_{\text {eye, } k}$ is the relative eye closure attributed to SPM/GVD and optical filtering effects, $\sigma_{A S E, k}^{2}$ is the electrical variance of ASE noise, while $\sigma_{F W M, k}^{2}$ and $\sigma_{X P M, k}^{2}$ are the electrical variance of FWM and XPM induced degradation respectively. Details on each one of these terms are provided next.

1) ASE noise: the electrical variance of the ASE noise [7] of the $k$-th link, calculated at the end of the cascade of the optical amplifiers, can be expressed as:

$$
\sigma_{A S E, k}^{2}=4 R^{2} P_{a v g} P_{A S E} \cdot \frac{B_{e}}{B_{o}},
$$

where $R$ is the responsivity of the receiver, $P_{a v g}$ is the average signal power at the receiver, $P_{A S E}$ is the ASE power of EDFAs [7], $B_{o}$ and $B_{e}$ are the optical and the electrical bandwidth of the receiver, respectively.

2) $S P M / G V D$ : both SPM and GVD are responsible for the broadening of the pulse. For this reason their effect can be combined and considered together[14][15]. By convoluting the transfer function of the fiber with the assumed frequency chirping at the transmitter, the eye closure penalty ( pen $_{\text {eye }, k}$ ) due to SPM/GVD at the end of the link can be calculated as:

$$
\text { pen }_{\text {eye }, k}=A_{\text {chirpped }}(\omega) * H_{\text {fiber }}(\omega) \text {, }
$$

where $A_{\text {chirpped }}(\omega)$ is the transmitted pulse with frequency chirping (modeled as in [6][7][15]), and $H_{\text {fiber }}(\omega)$ is the transfer function of the fiber.

3) FWM: the electrical variance of FWM of the $k$-th link can be obtained by using the following expression [16]:

$$
\sigma_{F W M, k}^{2}=2 R^{2} P_{t}\left(\frac{1}{8} \sum_{I} P_{a b c}+\frac{1}{4} \sum_{I I} P_{a b m}+\frac{1}{4} \sum_{I I I} P_{a a c}\right),
$$

where $R$ is the responsivity of the receiver, $P_{t}$ is the transmitted signal power of each channel, the first summation term represents the FWM power [17] in the case that all channels including the considered channel $(m)$ are using different frequencies $\left(f_{a} \neq f_{b} \neq f_{c} \neq f_{m}\right)$, the second summation represents the FWM power when $f_{a} \neq f_{b} \neq f_{c}$ but $f_{c}=f_{m}$, and the last summation represents the FWM power when $f_{a}=f_{b} \neq f_{c} \neq f_{m}$.

4) XPM: The electrical variance of XPM of the $k$-th link can be expressed by [18],

$$
\begin{gathered}
\sigma_{X P M, k, a}^{2}=P^{2} \sum_{b=1, b \neq a}^{N} \frac{1}{2 \pi} \int_{-\infty}^{o}[(x) \cdot(y) \cdot(z)] d \omega \\
(x)=\left|H_{X P M, a b}^{I M}(\omega)\right|^{2},(y)=\left|H_{\text {opt.filter }}(\omega)\right|^{2},(z)=P S D_{b}(\omega)
\end{gathered}
$$

where $P$ is the average channel power, $N$ is the total number of SMF spans, $H_{X P M, a b}^{I M}(\omega)$ is the XPM-induced intensity modulation frequency response originated by the pump channel $b$ on the probe channel $a, H_{\text {opt. filter }}(\omega)$ is the transfer function of the optical filter at the receiver, and $P S D_{b}(\omega)$ is the power spectral density of pump 
channel $b$. The detailed calculation of $H_{X P M, a b}^{I M}(\omega)$ can be found in [19].

\section{Estimating the transmission quality of a path in the network}

Once a connection request is routed along a given path, the resulting transmission quality needs to be assessed. A path $p \in G=(N, L)$ is assumed to be a sequence of network link, where the Q-penalty factor of each link is expressed as in Equation (1). The Q-factor of a path $p$ can be expressed as:

$$
Q_{p}=\frac{\text { pen }_{\text {eye }, p} \cdot P_{t}}{\sqrt{\sum_{k \in p} \sigma_{A S E, k}^{2}+\sum_{k \in p} \sigma_{F W M, k}^{2}+\sum_{k \in p} \sigma_{X P M, k}^{2}}}
$$

where $P_{t}$ is the transmitted signal power, pen $n_{\text {eye }, p}$ is the eye closure penalty due to SPM/GVD effects (as defined in Equation (3)) calculated basing on the total length of the path, $\sum_{k \in p} \sigma_{A S E, k}^{2}$ is the total electrical variance of ASE noise (as defined in Equation (2)) of the path, $\sum_{k \in p} \sigma_{F W M, k}^{2}$ and $\sum_{k \in p} \sigma_{X P M, k}^{2}$ are the total noise variance of FWM and XPM of the path (as defined in Equation (4) and (5)), respectively.

The BER value of a connection request routed along $p$ can be derived from Equation (6) as follows:

$$
B E R_{p}=\frac{1}{2} \operatorname{erfc}\left(\frac{Q_{p}}{\sqrt{2}}\right)
$$

\section{ICBR-DIFF: AN IMPAIRMENT CONSTRAINT BASED} ROUTING ALGORITHM WITH SERVICE DIFFERENTIATION

In this section, the general idea of ICBR-Diff algorithm is presented, followed by a detailed description of the algorithm.

Let $R=\left\{r_{1}, r_{2}, \ldots, r_{q}\right\}$ represent the set of connection requests that needs to be provisioned in $G=(N, L)$. The source and destination nodes of $r_{i} \in R$ are denoted by $s\left(r_{i}\right)$ and by $d\left(r_{i}\right)$ respectively.
It is assumed that the connection requests in $R$ are grouped in different classes, $\left\{C_{1}, C_{2}, \ldots, C_{v}\right\}$, where each class corresponds to a different BER threshold according to their signal quality requirements. Also, the assignment of connection class $C_{i}$ occurs in a one-to-one basis. Let $P^{i, j}=\left\{p_{1}^{i, j}, p_{2}^{i, j}, \ldots, p_{m}^{i, j}\right\}$ represent the set of $m$ candidate routes between source node $i$ and destination node $j$, and $B E R^{P^{i, j}}=\left\{B E R_{p_{1}^{i, j}}, B E R_{p_{2}^{i, j}}, \ldots, B E R_{p_{m}^{i, j}}\right\}$ represent the BER (computed as in Equation (7)) of each of the candidate routes in $P^{i, j}$.

Now, assume that $\bar{p} \in P^{r_{i}}$ is a candidate route for routing $r_{i}$. With any conventional ICBR approach, the BER of $\bar{p}$ (denoted as $\overline{B E R}$ ) is compared against a single and predefined BER threshold (denoted as $B E R^{\text {thrs }}$ ). This threshold, usually very stringent (e.g. $\left.10^{-15}, 10^{-16}\right)$, is chosen a priory and is used to check the feasibility of all $r_{i} \in R$. During this process, no attention is paid on what is the actual difference, in terms of BER, between $B E R^{\text {thrs }}$ and $\overline{B E R}$. As such, this approach may potentially lead to over-provisioning of network resources. The ICBR-Diff algorithm, on the other hand, tries to avoid over-provisioning of resources by trying to find a route $\bar{p}$ that (1) satisfies the signal quality requirement of $r_{i}$ (denoted as $B E R^{r_{i}}$ ) and, at the same time, (2) matches as closely as possible the value of $B E R^{r_{i}}$, i.e.,

$$
\begin{aligned}
\bar{p}=\min _{p_{j} \in P^{s\left(r_{i}\right), d\left(r_{i}\right)}}\left[B E R_{p_{j}^{s\left(r_{i}\right), d\left(r_{i}\right)}}-B E R^{r_{i}}\right] \\
\wedge\left[\left(B E R_{p_{j}^{s\left(r_{i}\right), d\left(r_{i}\right)}}-B E R^{r_{i}}\right)>0\right] .
\end{aligned}
$$

This in turn leads to a more efficient use of network resources (and consequently a lower blocking probability) as shown in the example that follows.

Fig. 2 shows an example where the ICBR-Diff algorithm and a conventional IABP algorithm are compared. Let $R=\left\{r_{1}, r_{2}, r_{3}\right\}$ be the set of connection requests from node $A$ to node $B$. Let also $10^{-15}, 10^{-9}$, and $10^{-15}$ be their respective signal quality requirements.

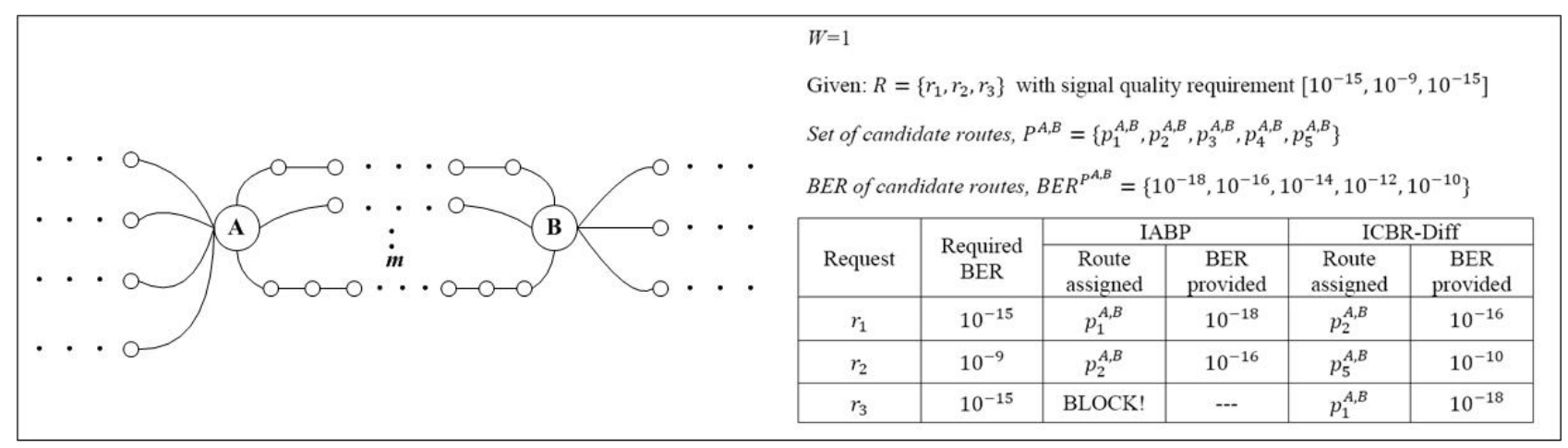

Figure 2. Path selection example. 


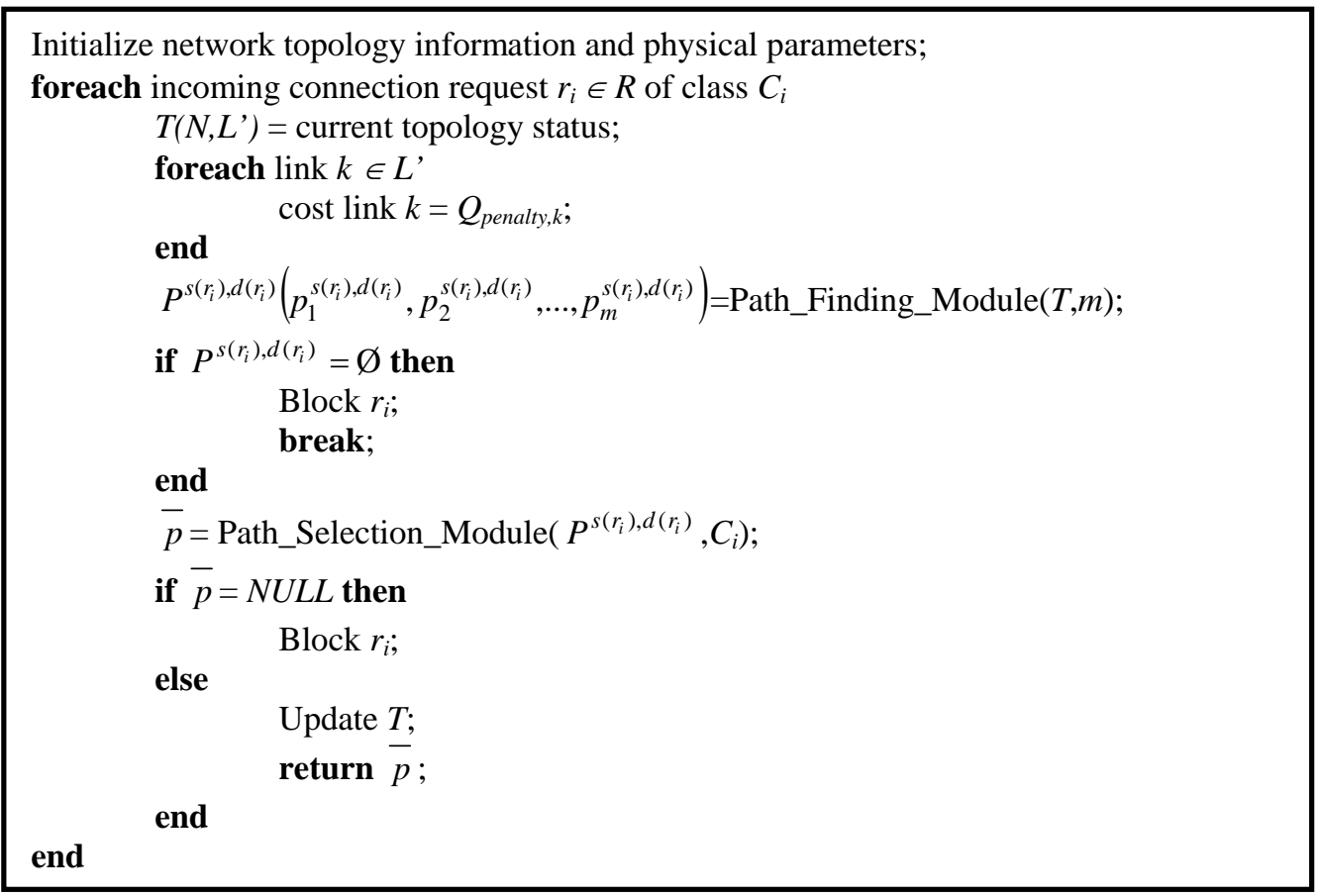

Figure 3. Pseudo code of ICBR-Diff.

We also assumed a specific assignment of routing requests to classes of service, namely: $\left\{r_{1}, r_{3}\right\} \in C_{1}$ with $\mathrm{BER} \leq 10^{-15}$ and $\left\{r_{2}\right\} \in C_{2}$ with $\mathrm{BER} \leq 10^{-9}$.

Let $P^{A, B}=\left\{p_{1}^{A, B}, p_{2}^{A, B}, p_{3}^{A, B}, p_{4}^{A, B}, p_{5}^{A, B}\right\}$ be the set of $m=5$ candidate routes between node $\mathrm{A}$ and node $\mathrm{B}$, with one wavelength available per route (i.e. $\mathrm{W}=1$ ). The set of $\mathrm{BER}$ values of each candidate route, i.e. $B E R^{P^{A, B}}$, is shown in Fig. 2. As previously explained, the IABP algorithm fixes the BER threshold, $B E R^{\text {thrs }}$, to a constant value (in the example $B E R^{\text {thrs }}=10^{-15}$ ), while the ICBRDiff uses the threshold $B E R^{C_{1}}=10^{-15}$ or $B E R^{C_{2}}=10^{-9}$, depending on the class, which the connection request being processed belongs to.

When $r_{1}$ arrives, IABP assigns route $p_{1}^{A, B}$ to $r_{1}$, i.e. the least impaired route that satisfies $B E R^{\text {thrs }}$. ICBRDiff, on the other hand, assigns $p_{2}^{A, B}$ which is the route with the highest BER value in $B E R^{P^{A, B}}$ that satisfies the signal quality requirement of the connection request of $C_{1}$. When $r_{2}$ arrives, IABP chooses $p_{2}^{A, B}$ while ICBRDiff selects $p_{5}^{A, B}$. Notice that $r_{2}$ belongs to $C_{2}$ and requires a BER value less than $10^{-9}$, while $p_{2}^{A, B}$ provides a BER value equal to $10^{-16}$. Finally, when $r_{3}$ arrives, IABP is not able to find a feasible route, since none of routes left with resources in $P^{A, B}$ satisfies the signal quality imposed by $B E R^{\text {thrs }}$. Instead, ICBR-Diff is able to assign $p_{1}^{A, B}$ to $r_{3}$. The rationale from the example is the following: ICBR-Diff avoids unnecessary blocking by selecting routes that closely match the connection request quality of service requirement.

The pseudo code of the ICBR-Diff algorithm is presented in Fig. 3. The algorithm starts with an initialization phase where the network topology information is collected, e.g. number of nodes, number of links, link lengths, link capacities, and all physical parameters required for the calculation of the Q-penalty factor of each link. Graph $T\left(N, L\right.$ '), with $L^{\prime} \subseteq L$, represents the current resource usage in the network, i.e. a link belongs to $L$ ' if and only if it has resources available. Initially, graph $T\left(N, L^{\prime}\right)$ is equal to graph $G(N, L)$. The algorithm assigns the Q-penalty factor, $Q_{\text {penalty, }}$, calculated as in Equation (1) as the cost for each link. Graph $T\left(N, L^{\prime}\right)$ is then weighted by using these link cost values. After assigning link costs, up to $m$ alternative routes for each connection request are computed by running the Dijkstra algorithm on the weighted graph $T(N, L ')$. Fig. 4 presents the pseudo code of the algorithm used to find $m$ alternative routes. If there is a route $p$ with at least one common wavelength available on every link, this route is stored in the set of candidate routes $P^{s\left(r_{i}\right), d\left(r_{i}\right)}$. The cost of links of $p$ is then doubled, the weight of the links in graph $T$ is updated with the new link costs, and additional candidate routes are computed. Otherwise, if no route is found or if there is not a common available wavelength on $p$, the connection request is blocked.

Next, the BER of each candidate route in set $P^{s\left(r_{i}\right), d\left(r_{i}\right)}$ is calculated using Equation (7). Fig. 5 shows the pseudo code of the path selection process implemented in the ICBR-Diff algorithm. With ICBR-Diff, different signal quality thresholds are considered according to the signal quality requirements of the incoming connection requests. 

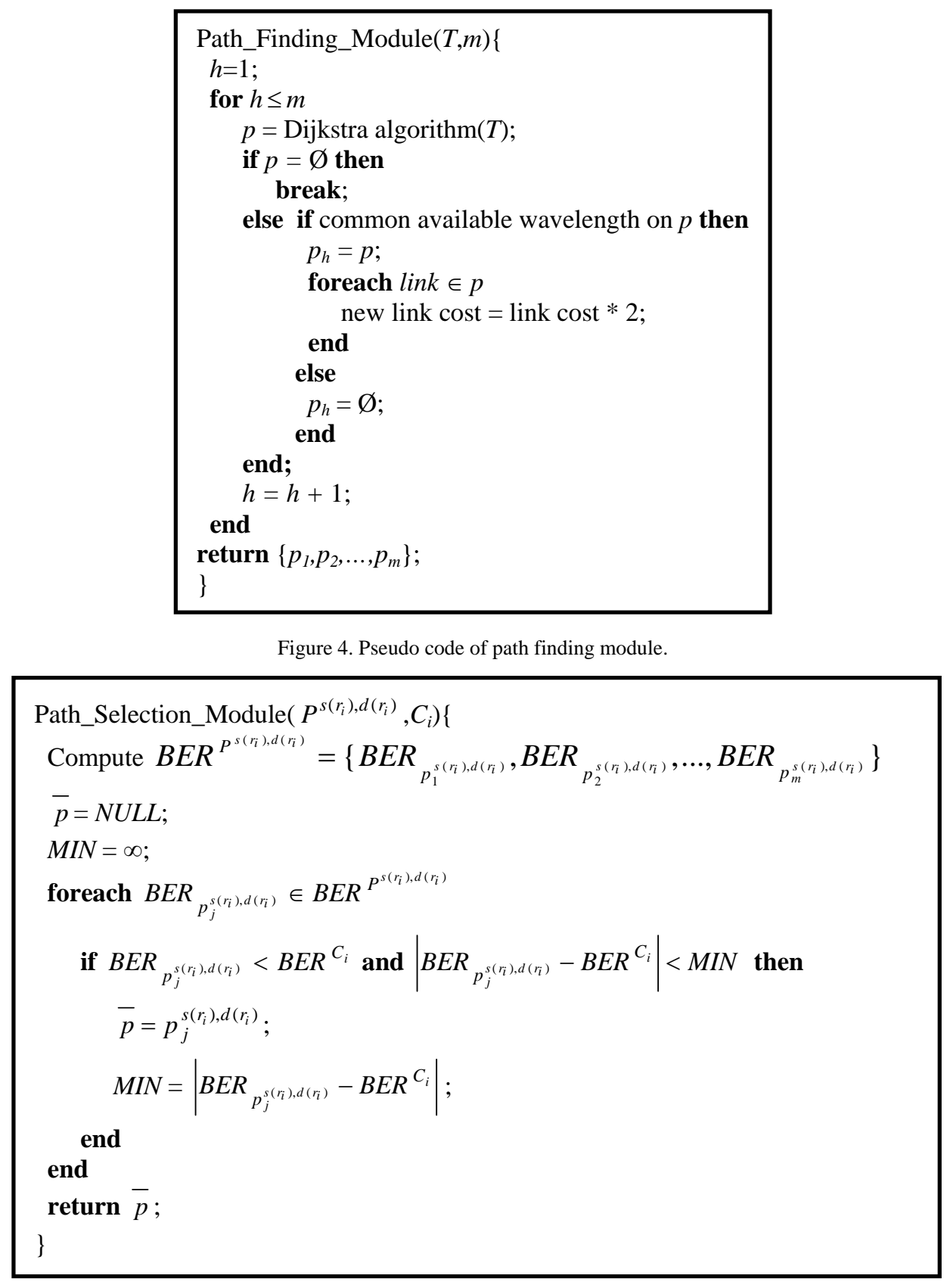

Figure 5. Pseudo code of path selection module.

When starting the path selection process (Fig. 5), the signal quality threshold is set to be the specific value based on the class the connection request belongs to. The BER of each candidate route $p_{j}^{s\left(r_{i}\right), d\left(r_{i}\right)}$ in set $P^{s\left(r_{i}\right), d\left(r_{i}\right)}$ is compared against the signal quality threshold of the class of the considered connection request (i.e. $B E R^{C_{i}}$ ). The candidate route $p_{j}^{s\left(r_{i}\right), d\left(r_{i}\right)}$ with the highest BER that satisfies the signal quality requirement of the connection request is selected. Finally, the first wavelength in the list of available wavelengths of the selected route is chosen to route the lightpath for the corresponding connection request.

\section{Performance Evaluation}

In this section, we first introduce the definitions and assumptions used in the evaluation of ICBR-Diff. Then some simulation results are presented.

\section{A. Definitions and assumptions}

It is assumed that the bandwidth demand of each connection request is one wavelength unit and that wavelength conversion capability is not available, i.e., wavelength continuity constraint is enforced while solving the routing problem. Furthermore, our experiment model assumes random and dynamic incoming connection requests that are sequentially served without prior knowledge of future incoming connection requests. 
TABLE I.

SYSTEM PARAMETERS USED FOR IMPAIRMENTS CALCULATION

\begin{tabular}{|l|c|}
\hline \multicolumn{1}{|c|}{ Parameters } & Values \\
\hline Central wavelength & $1553.6 \mathrm{~nm}(193.1 \mathrm{THz})$ \\
\hline Channel spacing & $50 \mathrm{GHz}$ \\
\hline Channel bit rate & $10 \mathrm{Gbit} / \mathrm{s}$ \\
\hline $\begin{array}{l}\text { Spontaneous-emission factor of } \\
\text { in-line EDFA }\left(n_{s p}\right)\end{array}$ \\
\hline $\begin{array}{l}\text { Spontaneous-emission factor of } \\
\text { EDFA before entering node }\left(n_{\text {sp }}\right)\end{array}$ & 2.5 \\
\hline Attenuation of SMF & $0.25 \mathrm{~dB} / \mathrm{km}$ \\
\hline Attenuation of DCF & $0.5 \mathrm{~dB} / \mathrm{km}$ \\
\hline $\begin{array}{l}\text { Nonlinear index coefficient of } \\
\text { SMF }\end{array}$ & $2.6 \times 10^{-20} \mathrm{~m}^{2} / \mathrm{W}$ \\
\hline $\begin{array}{l}\text { Nonlinear index coefficient of } \\
\text { DCF }\end{array}$ & $3.5 \times 10^{-20} \mathrm{~m}^{2} / \mathrm{W}$ \\
\hline GVD parameters of SMF & $17 \times 10^{-6} \mathrm{~s} / \mathrm{m}^{2}$ \\
\hline GVD parameter of DCF & $-80 \times 10^{-6} \mathrm{~s} / \mathrm{m}^{2}$ \\
\hline Dispersion slope of SMF & $0.085 \times 10^{3} \mathrm{~s} / \mathrm{m}$ \\
\hline Dispersion slope of DCF & $-0.3 \times 10^{-3} \mathrm{~s} / \mathrm{m}$ \\
\hline Effective area of SMF & $65 \times 10^{-12} \mathrm{~m}^{2}$ \\
\hline Effective area of DCF & $22 \times 10^{-12} \mathrm{~m}^{2}$ \\
\hline Optical bandwidth $\left(B_{o}\right)$ & $40 \mathrm{GHz}$ \\
\hline Electrical bandwidth $\left(B_{e}\right)$ & $7 \mathrm{GHz}$ \\
\hline Maximum length of SMF & $80 \mathrm{~km}$ \\
\hline
\end{tabular}

The incoming connection requests follow a Poisson distribution, while source/destination pairs are randomly chosen with equal probability (uniform distribution) among all network nodes. Connection holding time is exponentially distributed with the mean equal to 6 time units.

As mentioned earlier, ICBR-Diff supports differentiation of services, whereby connection requests are divided into two distinct classes with regard to their signal quality requirements, i.e. Class-1 connection requests that require higher signal quality in terms of maximum tolerated BER, and Class- 2 connection request that can tolerate higher signal degradation than Class- 1 . Throughout our simulations, Class- 1 connection requests require BER less than $10^{-15}$ and Class-2 connection requests require $\mathrm{BER}$ less than $10^{-9}$. Furthermore, two configurations of traffic mix for Class-1 and Class-2 connection request are considered: (i) $30 \%$ of the overall traffic being of Class-1, while $70 \%$ of the traffic being of Class-2 and (ii) equal share of Class-1 and Class-2, i.e. each class amounting to $50 \%$ of the overall traffic.

These signal quality requirements of Class- 1 and Class-2 connection requests were chosen based on available IP traffic measurement [20][21]. It is shown that streaming media traffic, i.e. traffic requiring higher signal quality (Class-1), accounts for much less of the total bandwidth utilization, whereas peer-to-peer (P2P) and World Wide Web (WWW) traffic, i.e. traffic requiring lower signal quality (Class-2), is dominating.

The parameter values used for the calculation of physical layer impairments in this work are listed in Table I.

\section{B. Simulation results}

To evaluate our ICBR-Diff algorithm, we used (i) the Pan-European test network topology (COST 239) [22], which comprises 11 nodes and 26 bidirectional fiber links with 16 wavelengths per fiber, and (ii) the NSF network
(NSFNet) consisting of 16 nodes and 24 bidirectional fiber links with 16 wavelengths per fiber (Fig. 6).

For benchmarking purpose, we also evaluate two other provisioning algorithms, namely shortest path and IABP. In the shortest path algorithm, the physical link distance is used as the link cost. The candidate route with the shortest physical distance in the set $P$ is selected first, then the BER of the selected route is calculated against a single signal quality threshold ( $B E R^{\text {thrs }}$ ). With IABP, each link is assigned a cost equal to $Q_{\text {penalty, } k}$, and the BER of every candidate route in the set $P$ is calculated and compared against a single signal quality threshold, $B E R^{\text {thrs }}$. The candidate route with the lowest BER value that satisfies the single signal quality threshold is selected. For both shortest path and IABP, the value of $B E R^{\text {thrs }}$ is set to be equal to $10^{-15}$. These two approaches do not support service differentiation and thus connection requests are blocked if there is no route with BER less than $B E R^{\text {thrs }}$, irrespectively of the class that the connection request belongs to. In the case of ICBR-Diff algorithm, Class- 1 connection request is blocked if there is no available lightpath connecting source and destination which exhibits BER less than $10^{-15}$, whereas Class-2 connection request is blocked if there is no lightpath with BER less than $10^{-9}$.

The total blocking probability shown in Fig. 7 accounts for both blocking due to insufficient resources, i.e. no wavelength is available, and due to the impairment constraints, when the candidate routes cannot meet the signal quality requirement. Additionally, the probability of connection blocking due to insufficient resources and the probability of connection blocking due to impairments are separately showed in Fig. 8 and Fig. 9, respectively. In both COST 239 and NSFNet topologies, the results show significant improvement in terms of connection blocking achieved by the ICBR-Diff algorithm, compared to shortest path and IABP routing. When Class- 1 and Class- 2 connection requests account for the $30 \%$ and $70 \%$ of total requests respectively, the benefit obtained by our ICBR-Diff algorithm in COST 239 topology is almost up to an order of magnitude in terms of total connection blocking compared to shortest path routing, and up to $81 \%$ compared to IABP algorithm, While the benefit achieved by ICBR-Diff in NSFNet is up to $61 \%$, compared to both shortest path and IABP approaches, since the connection blocking in the case of IABP is almost identical with the connection blocking in the case of shortest path algorithm. In the second case considering a traffic mix of Class-1 and Class-2 connection requests being equally weighted, the benefit reduces to $89 \%$ and $67 \%$ in the case of COST 239, and to $45 \%$ in the case of NSFNet.

By comparing the connection blocking in the case of shortest path routing against the IABP, we can see that the connection blocking reduction achieved by IABP algorithm is significant in the COST 239 topology, while the connection blocking of shortest path routing and IABP algorithms are almost identical in the NSFNet 

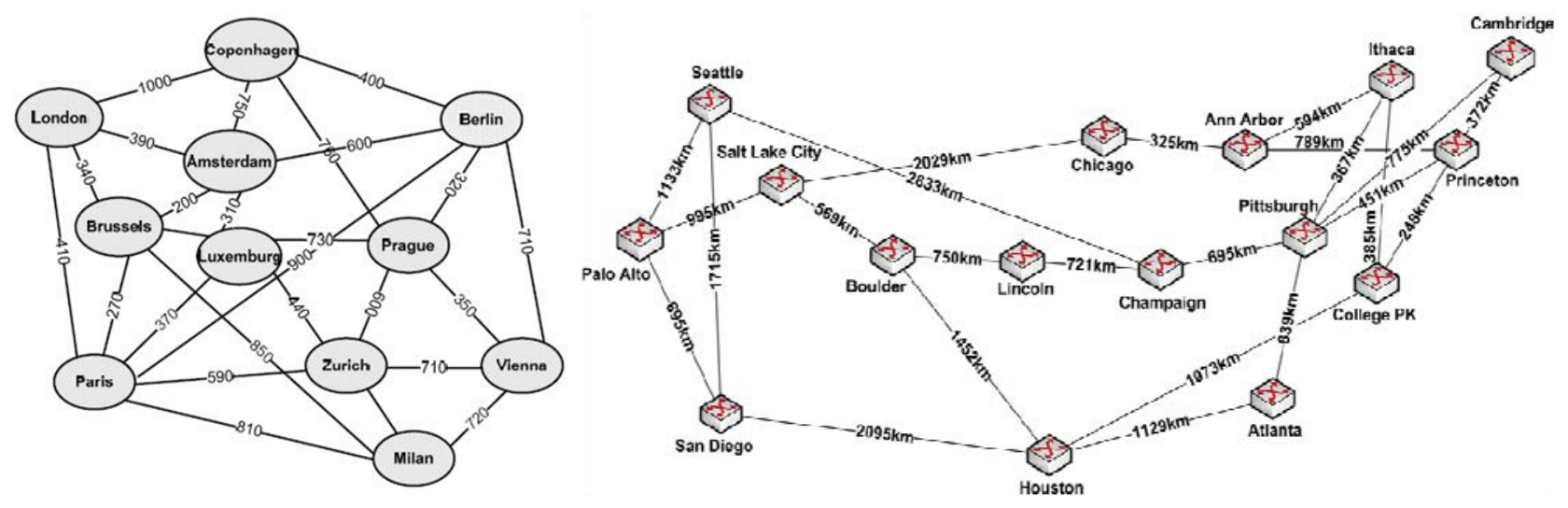

Figure 6. Pan-European COST 239 test network (left), and NSF network (right).
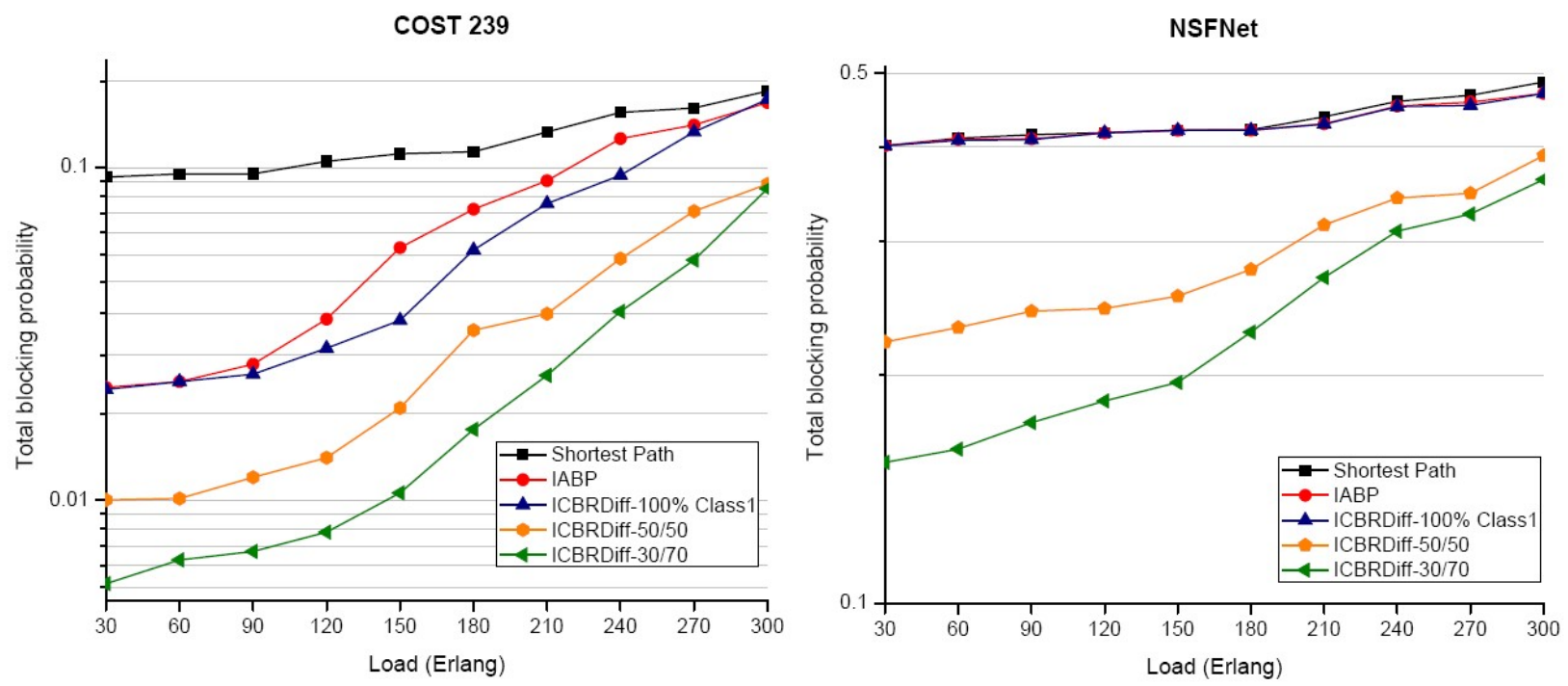

Figure 7. Total blocking probability versus load.
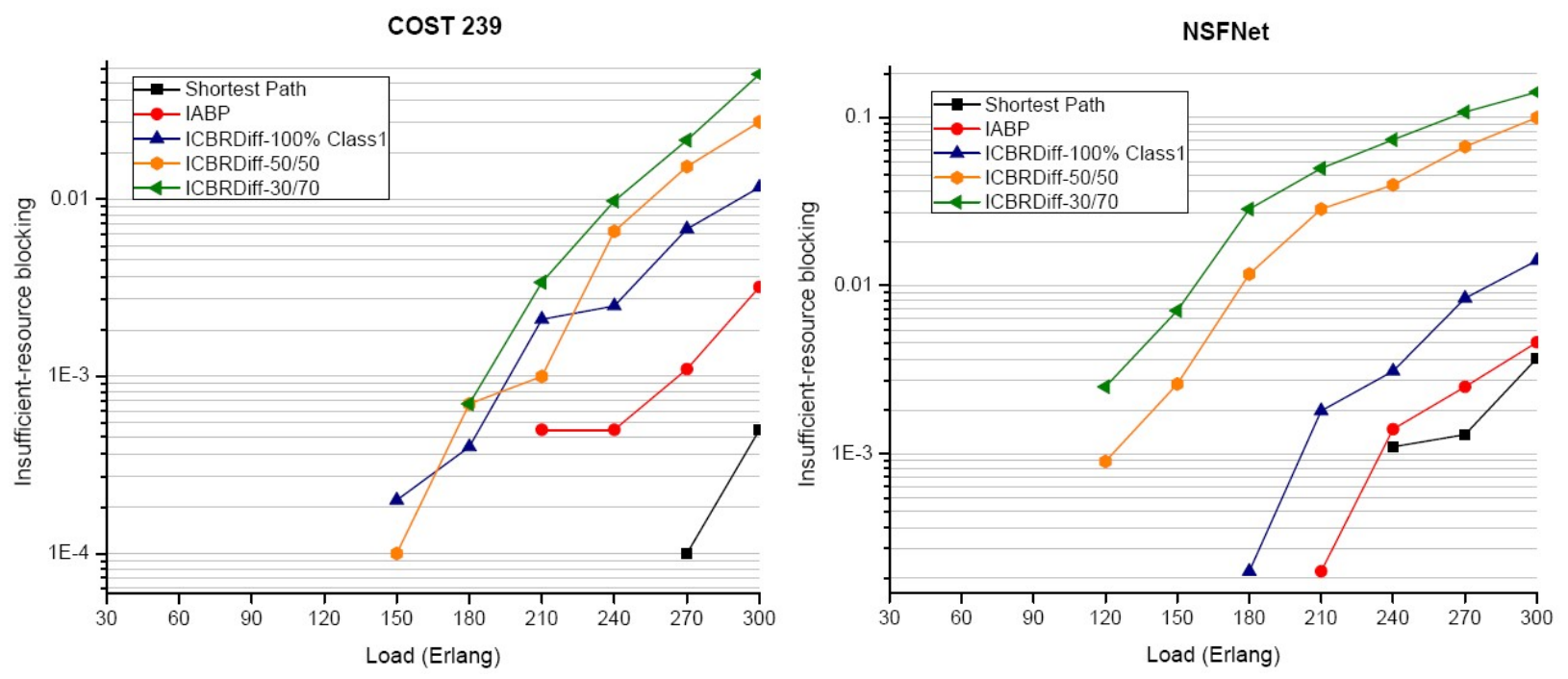

Figure 8. Insufficient-resource blocking versus load. 

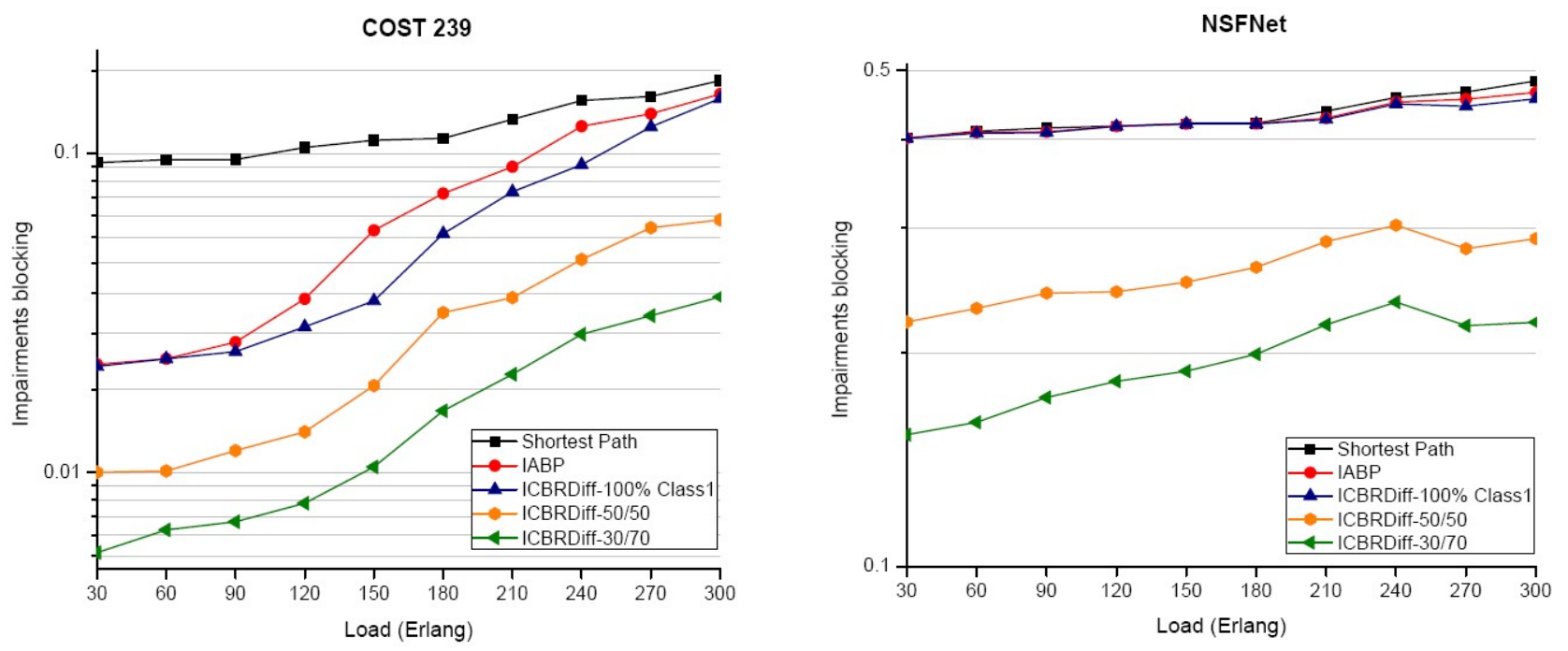

Figure 9. Impairments blocking versus load.

topology. This is because the diversity of the set of candidate routes is higher in COST 239 than in NSFNet, due to the higher nodal degree (see Table II and Fig. 6). Thus, the benefit, in terms of connection blocking, achieved by IABP in COST 239 is larger than that in NSFNet. Furthermore, the link length in COST 239 is relatively short, which in turns means the less impaired link. Thus, in IABP, there might be the possibility that a candidate route which is not the shortest one, in terms of physical length, is able to satisfy the signal quality requirement of the connection request. By considering all candidate routes rather than choosing the shortest one first, i.e. the shortest distance, IABP already gives the performance improvement in terms of connection blocking compared to shortest path routing. However, in ICBR-Diff, a higher improvement in connection blocking can be achieved by both considering all candidate routes and providing only the sufficient-signal quality lightpaths to the connection requests.

Fig. 7 shows that in COST 239 a slight performance improvement is gained by our ICBR-Diff, i.e. ICBRDiff$100 \%$ Class-1 in the figure, compared to IABP algorithm. To further improve the connection blocking, differentiation among connection requests is applied, and the simulation results (Fig. 7) show that significant performance improvement in terms of connection blocking can be achieved using the proposed ICBR-Diff algorithm.

In the case of NSFNet where the diversity of the set of the candidate routes is low due to the low nodal degree and the link length is relatively long compared to COST 239, shortest path routing and IABP end up selecting the same route. Because of the long link length, the route that satisfies the signal quality threshold is the shortest route also. By providing only the good-enough lightpath without the consideration of the differentiation of connection requests, i.e. ICBRDiff- $100 \%$ Class-1, ICBRDiff gives similar performance compared to IABP. This is also because of the low diversity of the set of the candidate routes and the long link length in NSFNet. However, when considering also the differentiation of
TABLE II.

COMPARISION BETWEEN COST 239 AND NSFNET

\begin{tabular}{|c|c|c|}
\hline Topology & COST 239 & NSFNet \\
\hline Number of nodes & 11 & 16 \\
\hline Number of bidirectional links & 26 & 24 \\
\hline Average node degree & 4.73 & 3 \\
\hline
\end{tabular}

signal quality requirements of connection requests during the connection provisioning phase, the considerably connection blocking improvement can be obtained by our ICBR-Diff algorithm.

The comparison between the simulation results of COST 239 and NSFNet shows that the ICBR-Diff algorithm is able to facilitate improved utilization of the network resources in both high and low nodal degree topologies, i.e. COST 239 and NSFNet respectively, compared to shortest path routing and the IABP approach. Moreover, in the low nodal degree and long link length topology, i.e. NSFNet, our ICBR-Diff algorithm can still significantly improve the performance in terms of connection blocking, while IABP cannot.

\section{CONCLUSIONS}

In this paper, we proposed a novel Impairment Constraint Based Routing (ICBR) algorithm with differentiation of services based on the BER of a lightpath for each connection request. In contrast to the existing ICBR and Impairment-Aware Best-Path (IABP) algorithms, in our approach the signal quality requirement of the connection request, in terms of maximum tolerated BER, is considered as a routing constraint during the connection-provisioning phase. Simulation results indicate significant improvement in connection blocking in both COST 239 and NSFNet compared to shortest path routing and the IABP approach. By assigning lightpaths to the connection requests, having acceptable BER performance with respect to the predefined thresholds, and by avoiding to choose lighpaths with a lower BER value compared to the threshold required, our ICBR-Diff algorithm is able to offer more efficient resource utilization. This is due to 
that the best performing lighpaths remain available for future use when new and more demanding connection requests, in terms of BER, are to be set up. Furthermore, another interesting finding is that our ICBR-Diff has a high flexibility to different network topologies.

The network performance improvement offered by the proposed ICBR-Diff has been obtained by avoiding connection blocking due to unnecessary high signal quality constraint.

Simulation results show that in the topology where the node degree is low, i.e. the diversity of the set of the candidate routes is low, and the link length is long (NSFNet), our ICBR-Diff is still able to give significant performance improvement in terms of connection blocking, while IABP gives similar performance to shortest path routing.

\section{ACKNOWLEDGMENT}

This work was supported by the Network of Excellence "Building the Future Optical Network in Europe” (BONE), funded by the European Commission through the $7^{\text {th }}$ ICT-Framework Programme and the EUREKA/CELTIC project "Management Platform for Next Generation Optical Networks” (MANGO).

\section{REFERENCES}

[1] I. Chlamtac, A. Ganz, and G. Karmi, "Lightpath Communications: a novel approach to high bandwidth optical WANs”, IEEE Transactions on Communications, vol. 40, no. 7, pp. 1171-1182, 1992.

[2] J. Berthold, A.A.M. Saleh, L. Blair, and J.M. Simmons, "Optical Networking: past present and future", IEEE/OSA Journal of Lightwave Technology, vol. 26, no. 9, pp. 11041118, 2008.

[3] R. Ramasawami and K.N. Sivarajan, "Routing and Wavelength Assignment in All-Optical Networks", IEEE/ACM Transaction on Networking, vol. 3, no. 5, pp. 489-500, Oct. 1995.

[4] D. Cavendish, A. Kolarov, and B. Sengupta, "Routing and Wavelength Assignment in WDM Mesh Networks", in Proc. Conf. Globecom 2004, Dec. 2004.

[5] J. Strand, A.L. Chiu, and R. Tkach, "Issues For Routing In The Optical Layer", IEEE Communications Magazine, vol. 39, no. 2, pp. 81-87, Feb. 2001.

[6] R. Ramasawami, and K.N. Sivarajan, "Optical networks: a practical perspective”, $2^{\text {nd }}$ edition, Morgan Kaufmann, San Francisco, 2002.

[7] G.P. Agrawal, "Fiber-Optic Communication Systems", $3^{\text {rd }}$ edition, A John Wiley \& Sons, INC., Publication, New York, 2002.

[8] M. Farahmand, D. Awduche, S. Tibuleac, and D. Atlas, "Characterization and representation of impairments for routing and path control in all-optical networks", in Proc. of National Fiber Optic Engineers Conference (NFOEC), Dallas, TX, Sep. 2002.

[9] B. Ramamurthy, D. Datta, H. Feng, J.P. Heritage, and B. Mukherjee, "Impact of transmission impairments on the teletraffic performance of wavelength-routed optical networks”, IEEE/OSA Journal of Lightwave Technology, vol. 17, no. 10, pp. 1713-1723, Oct. 1999.

[10] Y. Huang, J.P. Heritage, and B. Mukherjee, “Connection Provisioning With Transmission Impairment Consideration in Optical WDM Networks With High-
Speed Channels", IEEE/OSA Journal of Lightwave Technology, vol. 23, no. 3, pp. 982-993, Mar. 2005.

[11] G. Markidis, S. Sygletos, A. Tzanakaki, and I. Tomkos, "Impairment Aware based Routing and Wavelength Assignment in Transparent Long Hual Networks", in Proc. Conf. on Optical Network Design and Modeling (ONDM), May 2007.

[12] D. Penninckx, G. Charlet, J.-C. Antona, and L. Noirie, "Simple engineering rules for a transparent wavebandbased optical backbone network", OSA Journal of Optical Networking, vol. 2, no. 2, pp. 38-45, Feb. 2003.

[13] I. Tomkos, M. Vasilyev, J.-K. Rhee, A. Kobyakov, M. Ajgaonkar, and M. Sharma, "Dispersion map design for 10 $\mathrm{Gb} / \mathrm{s}$ ultra-long haul DWDM transparent optical networks”, in Proc. OECC'02, Yokohama, Japan, Jul. 2002.

[14] M.I. Hayee, and A.E. Willner, "Pre- and PostCompensation of Dispersion and Nonlinearities in 10-Gb/s WDM Systems”, IEEE Photonics Technology Letters, vol. 9, no. 9, pp. 1271-1273, Sep. 1997.

[15] M. Stern, J.P. Heritage, R.N. Thurston, and S. Tu, "SelfPhase Modulation and Dispersion in High Data Rate Fiber-Optic Transmission Systems”, IEEE/OSA Journal of Lightwave Technology, vol. 8, no. 7, pp. 1009-1016, Jul. 1990.

[16] K. Inoue, K. Nakanishi, K. Oda, and H. Toba, ”Crosstalk and Power Penalty Due to Fiber Four-Wave Mixing in Multichannel Transmissions", Journal of Lightwave Technology, vol. 12, no. 8, pp. 1423-1439, Aug. 1994.

[17] K. Inoue, "Four-Wave Mixing in an Optical Fiber in the Zero-Dispersion Wavelength Region”, IEEE/OSA Journal of Lightwave Technology, vol. 10, no. 11, pp. 1553-1561, Nov. 1992.

[18] S. Pachnicke, S. Spalter, J. Reichert, and E. Voges, "Analytical Assessment of the Q-factor due to CrossPhase Modulation (XPM) in Multispan WDM Transmission Systems”, in Proc. SPIE, vol. 5247, pp. 6170, Sep. 2003.

[19] A.V.T. Cartaxo, "Cross-Phase Modulation in Intensity Modulation-Direct Detection WDM Systems with Multiple Optical Amplifiers and Dispersion Compensators”, IEEE/OSA Journal of Lightwave Technology, vol. 17, no. 2, pp. 178-190, Feb. 1999.

[20] Traffic Measurements and Models in Multi-Service Networks project, Celtic project, "TRAMMS IP Traffic report no. 1, April 2008”, http://projects.celticinitiative.org/tramms/.

[21] Traffic Measurements and Models in Multi-Service Networks project, Celtic project, "TRAMMS IP Traffic report no. 3, June 2008”, http://projects.celticinitiative.org/tramms/.

[22] P. Batchelor et al., "Study on the implementation of optical transparent transport networks in the European environment-Results of the research project COST 239", Photonic Network Communications, vol. 2, no. 1, pp. 1532, 2000.

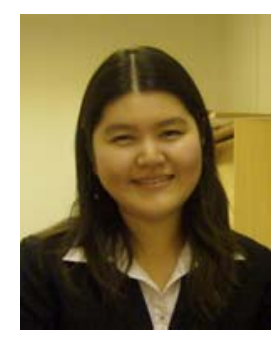

Amornrat Jirattigalachote obtained her Bachelor's degree in Telecommunication Engineering from King Mongkut's Institute of Technology Ladkrabang, Bangkok, Thailand in 2004, and her Master degree in Electrical Engineering (specialized in Photonics) from the Royal Institute of Technology (KTH), Stockholm, Sweden in 2008. 
She worked as a Tooling Software Development Engineer at Western Digital (Thailand) Co., Ltd. from 2004 to 2005. In 2005, she started working as a System Engineer with Aeronautical Radio of Thailand Ltd. From 2008, she is a Ph.D. student at KTH in the area of Optical Networking. Her main research interests presently are on Physical Layer Impairment Aware Routing in Multi-domain Multi-granularity Optical Networks and Power Efficiency in Survivable WDM Networks.

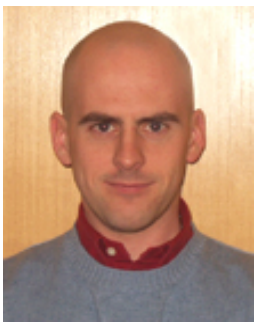

Paolo Monti received a Laurea degree in Electrical Engineering (2001) from the Politecnico di Torino, Italy, and a Ph.D. in Electrical Engineering (2005) from the University of Texas at Dallas (UTD).

From 2006 to 2008 he worked as a Research Associate of the Open Networking Advance Research (OpNeAR) Lab at UTD. He joined the Royal Institute of Technology (KTH) in September 2008 where he is currently an Assistant Professor in the School of Information and Communication Technology (ICT/FMI) and a member of the Next Generation Optical Networks (NEGONET) group. He co-authored more than thirty papers published in international journals and presented in leading international conferences. His research interests include network planning, protocol design, performance evaluation and optimization techniques for both optical and wireless networks.

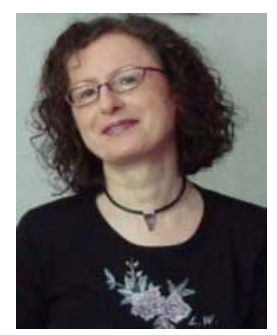

Lena Wosinska received her Ph.D. degree in photonics and the Docent degree in optical networking from the Royal Institute of Technology (KTH), Stockholm, Sweden, in 1999 and 2008, respectively.

She joined KTH in 1986, where she is currently an Associate Professor with the School of Information and Communication Technology (ICT), heading a research group in optical networking (Next Generation Optical Networks NEGONET) and coordinating a number of national and international scientific projects. Her research interests include optical network management, reliability and survivability of optical networks, photonics in switching, and fiber access networks.

Dr. Wosinska has been involved in a number of professional activities, including Guest Editorship of the following special issues that appeared in the OSA Journal of Optical Networking: High Availability in Optical Networks; Photonics in Switching; Reliability Issues in Optical Networks; and Optical Networks for the Future Internet. Since 2007, she has been an Associate Editor of the OSA Journal of Optical Networking, and since April 2009 she serves in the Editorial Board of the IEEE/OSA Journal of Optical Communications and Networking. Since 2005, she has been a General Chair of the Workshop on Reliability Issues in Next Generation Optical Networks (RONEXT), which is a part of the IEEE International Conference on Transparent Optical Networks (ICTON). She serves on the technical program committees of many international conferences.

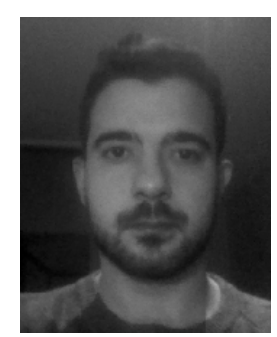

Kostas M. Katrinis earned the diploma in Computer Engineering from the Computer Engineering and Informatics Dept., University of Patras, Greece in 2000 and the Ph.D. in Technical Sciences from the Swiss Federal Institute of Technology (ETH Zurich), Zurich, Switzerland in 2006.

$\mathrm{He}$ is currently with the Network Design and Services (NDS) group at the Athens Information Technology (AIT), Athens, Greece. In the past, he has worked as a research assistant at the Communication Systems Group at ETH Zurich in the area of multimedia communication. He has authored or co-authored numerous publications in international journals and conferences and two book chapters. His research interests are in the area of network planning, sustainable networking, trustworthy communication and network performance evaluation.

Dr. Katrinis is a member of the Technical Chamber of Greece since 2000.

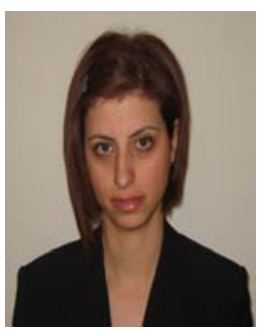

Anna Tzanakaki is an Associate Professor at the Athens Information Technology, where she is leading the Network Design and Services research group. She is also an adjunct faculty member of Carnegie Mellon University, USA. She has obtained a BSc degree from the University of Crete, Greece, an MSc and a PhD both from the University of Essex, UK. She was a co-founder and a senior engineer of ilotron ltd, a spin-off from the University of Essex, involved in the design of systems for WDM optical networks. Following ilotron, she joined Altamar Networks, a subsidiary of Ditech Communications, as a principal engineer responsible for optical architecture and system design. She is a co-author of over 100 publications in international journals and conferences. She is a co-inventor of 1 granted and 11 published patents. She is a senior member of the IEEE and several Technical Program Committees. Her research interests include optical wavelength, burst and packet switched networks, cross-layer network design and traffic provisioning as well as network convergence in support of telecommunications and IT services. 